\title{
Deposition of powdered activated carbon (PAC) on ultrafiltration (UF) membrane surface: influencing factors and mechanisms
}

Senlin Shao ${ }^{a}$, Luyang Cai ${ }^{a}, \mathrm{Kai}_{\mathrm{Li}}{ }^{b}$, Jiangyun $\mathrm{Li}{ }^{*}{ }^{a}$, Xing $\mathrm{Du}{ }^{c}$, Guibai $\mathrm{Li}^{c}$, Heng Liang ${ }^{*} c$

${ }^{a}$ School of Civil Engineering, Wuhan University, Wuhan, Hubei, 430072, PR China

${ }^{b}$ School of Environmental and Municipal Engineering, Xi'an University of Architecture and Technology, 13 Yanta Road, Xi'an 710055, PR China

${ }^{c}$ State Key Laboratory of Urban Water Resource and Environment (SKLUWRE),

Harbin Institute of Technology, 73 Huanghe Road, Nangang District, Harbin 150090, PR China

E-mail address

shaosenlin@gmail.com (S.Shao); luyangcaiwhu@163.com(L.Cai);

likai02007@126.com (K.Li); lijy@whu.edu.cn (J.Li); hitduxing@163.com (X.Du);

liguibai@vip.163.com (G. Li); hitliangheng@163.com (H. Liang).

*Corresponding Authors

Tel./fax: +86 451 86283001; E-mail: hitliangheng@163.com (H. Liang) 


\section{Abstract}

The deposition of powdered activated carbon (PAC) on an ultrafiltration (UF) membrane surface, a common phenomenon in the PAC/UF hybrid process, may have a pronounced influence on UF operation. In this study, the influencing factors and mechanisms of the deposition of PAC were systematically investigated. The influencing factors included the organic species, membrane material, PAC size, filtration volume, and cleaning water. The experimental results suggested that PAC itself was rarely deposited on the membrane surface, forming a monolayer. However, in the cases when organic matter was heavily deposited on the membrane surface (e.g., humic acid (HA) and sodium alginate (SA) in the presence of $0.5 \mathrm{mmol} / \mathrm{L}$ calcium ions), multilayers of PAC and the organic matter formed; thus, the mass of the deposited PAC significantly increased. The deposition of PAC was barely affected by the membrane material, and decreased as the PAC size increased. Physical cleaning with pure water could effectively detach the deposited PAC. The HA adsorbed by the PAC and the membrane hindered the deposition of PAC because this part of the HA decreased the attractive force between the PAC and the membrane. However, the HA rejected by the membrane promoted the deposition of PAC, as evidenced by the fact that the mass of the deposited PAC increased as the mass of the deposited HA increased. The PAC could be "trapped" in the layer of HA when it formed a cake layer with the HA rejected by the membrane.

Key words: ultrafiltration (UF), powdered activated carbon (PAC), deposition, 
cake layer.

\section{Introduction}

Ultrafiltration (UF) coupled with powdered activated carbon (PAC) adsorption in integrated configuration (PAC/UF hybrid process) is a proven process to remove organic matter (e.g., natural organic matter, micropollutants, taste and odors) and particulate contaminants (e.g., Cryptosporidium) [1,2]. This process offers many advantages, such as a high efficiency, a small footprint, and flexible operation [3]. Although there are many bench- and pilot-scale studies of the PAC/UF hybrid process [4-8], there are no full-scale applications [2]. The main reason for this plight is that PAC is in contact with the membrane in the PAC/UF hybrid process, but PAC is not tailor-made to match the requirements of UF operation; thus, the contact between the PAC and the membrane possibly undermines the performance of UF [2]. In practical applications, to avoid contact between the PAC and the membrane, UF coupled with PAC adsorption process is preferentially built using a separated configuration (PAC-UF process); however, this configuration has a relatively large footprint and is costly [2]. By contrast, the PAC/UF hybrid process is effective, compact, and theoretically feasible. To promote the full-scale application of the PAC/UF hybrid process, it is crucial to understand how the PAC influences the operation of UF when PAC is in contact with membrane.

In the PAC/UF hybrid process, PAC has complex influences on the operation of UF. For example, our previous studies demonstrated that PAC has a double-edged 
effect on membrane fouling. On the one hand, PAC reduces the amount of organic matter deposited on the membrane surface $[6,9]$; on the other hand, PAC and the deposited organic matter have a synergistic effect on membrane fouling when they form a cake layer together [10]. In addition to these influences, PAC can be irreversibly deposited on the UF membrane surface; the deposited PAC is difficult to remove even by backwashing or bubble scouring (Fig. S1) [3, 11-15]. This current study mainly focused on the deposition of PAC on the UF membrane surface; the term “deposition" refers to PAC irreversibly deposited on the UF membrane, i.e., remains on the membrane surface after physical cleaning (e.g., cross flow, bubble scouring, and backwashing).

The deposition of PAC on the membrane surface may undermine the performance of UF in the following ways: (1) decreases the removal efficiency of pollutants by decreasing the effective concentration of PAC in the bulk liquid [3]; (2) increases membrane fouling by the synergistic effect of the deposited PAC and organic matter $[10,13]$; (3) facilitates biofouling by promoting the growth of bacteria (PAC is a good carrier for bacteria) $[11,16]$; (4) increases the risks of membrane breakage by increasing the tension force on the membrane; (5) decreases the chemical cleaning efficiency by increasing the chlorine demand [2].

Among the previous studies of the deposition of PAC, it is commonly believed that the organic-laden PAC is more easily deposited on the membrane surface $[15,17$, 18] because organic matter can serve as a "glue" for the deposition of PAC [19]. In 
addition to organic matter, metal ions may also facilitate the deposition of PAC [18]. Meanwhile, the operating parameters greatly affect the deposition of PAC. For example, decreasing flux, increasing cross-flow velocity, and prolonging hydraulic retention time (HRT) generally result in a reduction of deposited PAC $[3,20]$. Nevertheless, influencing factors, such as membrane material, organic species, and cleaning water have not yet been investigated. Although the previous publications assumed that the organic matter could "glue" the PAC on the membrane surface, there has been no definitive evidence or detailed investigation of this phenomenon. We still do not know how the PAC is "glued" onto the membrane surface. Therefore, the underlying mechanisms for the deposition of PAC also need to be further elucidated.

In studies of membrane fouling, the use of model substances enables a fundamental understanding of the fouling mechanism under well-defined conditions [21]. Humic acid (HA), bovine serum albumin (BSA), and sodium alginate (SA) are three model organic foulants that are typically used [22, 23]; they have also been used in our previous studies $[6,10]$. In the deposition of PAC, interactions among PAC, organic matter, and membrane are involved. The extended Derjaguin, Landau, Verwey and Overbeek (XDLOV) theory is generally used to interpret the interfacial interactions between a membrane and particles [23]. For example, the theory has been used to investigate the impact of the surface energy of the particles on membrane fouling ( $10 \mu \mathrm{m}$ polystyrene and glass particles were used) [24]. The theory was also used to determine the interaction forces between nanofiltration membranes and $2 \mu \mathrm{m}$ 
micro-beads [25].

The main objective of this study was to gain a better insight into the deposition of PAC on a UF membrane surface. The deposition of virgin PAC on the membrane surface was investigated first, and then the cases in which PAC could be heavily deposited on the membrane surface were identified using model organic foulants (HA, BSA, and SA). Subsequently, influencing factors for the deposition of PAC, including membrane material, PAC size, filtration volume, and cleaning water characteristics were studied. Finally, free adhesion energy analysis and scanning electronic microscopy (SEM) were used to examine and discuss the mechanisms involved in the deposition of PAC.

\section{Methods and Materials}

\subsection{Organic matter and PAC}

The HA, BSA, and SA were all from Sigma-Aldrich (St. Louis, MO, USA) and were used as representatives of humic substances, proteins, and polysaccharides, respectively. Stock solutions of the three types of organic matter were prepared according to the method described by Li et al. [6] and were stored in the dark at $4{ }^{\circ} \mathrm{C}$. The concentrations of HA, BSA, and SA solutions used in the experiment were 10, 2, and $2 \mathrm{mg} / \mathrm{L}$, respectively. A concentration of $1 \mathrm{mmol} / \mathrm{L} \mathrm{NaHCO}_{3}$ was added to the solution as a buffer, and the $\mathrm{pH}$ was adjusted to 7.5. The concentration of calcium ion in the solutions was either 0 or $0.5 \mathrm{mmol} / \mathrm{L}$. The ionic strength of the solutions was adjusted to $10 \mathrm{mmol} / \mathrm{L}$ using $\mathrm{NaCl}$. All organic matter solutions were filtered through 
a $0.45 \mu \mathrm{m}$ cellulose ester membrane (Taoyuan, China) prior to use.

The PAC used in this study has been previously described [10]. Briefly, a wood-based PAC (Bench Chemicals, China) was used. The Brunauer-Emmett-Teller (BET) surface area and the average pore size of the PAC were $1219 \mathrm{~m}^{2} / \mathrm{g}$ and $2.2 \mathrm{~nm}$, respectively. To obtain PAC with $d_{50}$ values of $69.4,24.2$ and $12.7 \mu \mathrm{m}$, the PAC was sieved through 140, 200, and 800 mesh screens, respectively. Unless noted otherwise, PAC with a $d_{50}$ of $24.2 \mu \mathrm{m}$ was used in experiments. The PAC was dispersed using ultrasound before use.

\subsection{UF experimental setup}

All the UF experiments were operated in dead-end, constant-flux mode using a membrane test cell (Amicon 8400, Millipore Corp., USA). During filtration, a peristaltic pump was used to maintain the flux $\left(150 \mathrm{~L} /\left(\mathrm{m}^{2} . \mathrm{h}\right)\right)$, and a pressure sensor was used to monitor the trans-membrane pressure (TMP). The volume of the feed solution was $350 \mathrm{~mL}$. Three types of membrane (PES, CA, and PVDF) were used to investigate the effect of the membrane material on the deposition of PAC. The membrane area and the molecular weight cutoff of the three membranes were 41.8 $\mathrm{cm}^{2}$ and $100 \mathrm{kDa}$, respectively. Other information concerning the three membranes is presented in Table S1 of the Supplementary material. Unless noted otherwise, the PES membrane was used. All the membranes were washed with Milli-Q water before use.

\subsection{Experimental protocol}

The protocol of the experiment is as follows (Fig. 1). (1) PAC (50 mg/L) was 
added to a flask filled with a solution of organic matter, and the flask was shaken in a rotary shaker for $60 \mathrm{~min}$. (2) Next, $350 \mathrm{~mL}$ of the mixture was added to the filtration cell, and $300 \mathrm{~mL}$ of the mixture was filtered at a constant flux of $150 \mathrm{~L} / \mathrm{m}^{2} . \mathrm{h}$ without stirring. (3) After filtration, the loosely deposited PAC was removed by stirring at a speed of $\sim 500 \mathrm{rpm}$ for $2 \mathrm{~min}$ with the residual $50 \mathrm{~mL}$ of concentrate. (4) The membrane was taken out and the PAC on the membrane surface was carefully collected using a plastic brush while simultaneously being flushed with Milli-Q water. (5) The mass of the deposited PAC was quantified using a turbidimeter $(2100 \mathrm{~N}$, $\mathrm{HACH}, \mathrm{USA}$ ). According to a computational fluid dynamics (CFD) simulation, under a stirring speed of $500 \mathrm{rpm}$, the shear stress of the fluid on the membrane surface ranged from 0.024 to $9.59 \mathrm{~Pa}$, and the area-weighted average shear stress was $4.37 \mathrm{~Pa}$ (Fig. S2 of the Supplementary material).

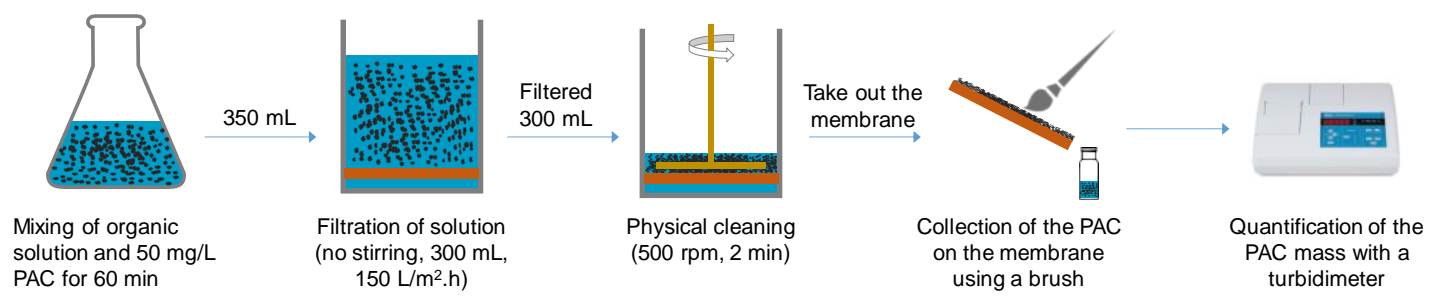

Fig.1 Experimental protocol for determining the mass of deposited PAC

\subsection{Analytical methods}

\subsubsection{The mass of PAC deposited on the membrane surface}

After physical cleaning, the foulants on the membrane surface were carefully scraped off using a plastic brush; the collected sample contained irreversibly 
deposited PAC and organic matter (i.e., HA, BSA, and SA). The BSA and SA had no influence on the turbidity of the solution, whereas HA increased the turbidity of the solution. Therefore, for the mixture of PAC and HA, the PAC should be separated before measurement. The mixture of PAC and HA was stirred for $24 \mathrm{~h}$ after adjusting its $\mathrm{pH}$ to 12 with $\mathrm{NaOH}$. It was subsequently centrifuged at $10000 \mathrm{rpm}$ for $10 \mathrm{~min}$, and the resulting PAC pellet was resuspended in Milli-Q water.

The PAC concentration and the solution turbidity showed a good linear relationship ( $R^{2}>0.99$, Fig. S3 of the Supplementary material). Therefore, the PAC concentration was quantified by the turbidity of the solution. The mass of the PAC could be acquired by multiplying the PAC concentration by the volume of the sample.

\subsubsection{Free energy of adhesion}

During membrane filtration, hydrodynamic forces bring PAC closer to the membrane surface, and then the short-range physicochemical interactions between PAC and the membrane determine the irreversible deposition of PAC [26]. The affinity between PAC and the membrane can be quantified using the free energy of adhesion, which shows the interaction energy per area between two surfaces that are brought into contact $[24,27]$. The free energy of adhesion was calculated according to the acid-base (van Oss) approach [26, 28]. Detailed information on the method can be found in the Supplementary material. In the method, contact angles of at least three liquids with known parameters are measured. The probe liquids used in this study

were diiodomethane (Sigma-Aldrich, USA), Milli-Q water and glycerol 
(Sigma-Aldrich, USA). The contact angle of the UF membrane was measured using a contact angle goniometer (JYSP-360, Beijing United Test Co., Ltd., China), according to the standard sessile drop method. The probe liquid at a volume of $2 \mu \mathrm{L}$ was dropped on the pre-dried membrane surface using a microsyringe. At least 8 measurements were performed on randomly selected locations for each sample. The contact angle of PAC was measured on the lawns of PAC deposited on an adhesive plaster. The PAC was uniformly coated on the adhesive plaster before measurement, which was indicated by the same water contact angles on randomly selected locations on the sample.

\subsubsection{Other analytical methods}

The HA concentration was determined at a wavelength of $254 \mathrm{~nm}$ using a UV/vis spectrophotometer (T6, Puxi, China). Turbidity was measured using a turbidimeter $(2100 \mathrm{~N}, \mathrm{HACH}, \mathrm{USA})$. The particle size of the PAC was measured using a Mastersizer 2000 (Malvern, UK). The zeta potential was determined using a Zetasizer NS (Malvern, UK).

SEM (Quanta 200, FEI, USA) was used to observe the surface morphologies of the membrane. Membrane samples were dried in an oven for 3-4 d, and the dried samples were then coated with gold using a precision etching coating system (Model 682, Gatan, USA) before SEM observation.

To statistically evaluate the effects of different factors on the deposition of PAC, analysis of variance (ANOVA) at the $p<0.05$ significance level was conducted using 
the Microsoft Excel software package (Microsoft Corporation, USA).

\section{Results and discussion}

\subsection{Deposition of PAC on the UF membrane with and without organic matter}

To gain a better insight into the deposition of PAC on the UF membrane surface, the deposition of PAC itself on the UF membrane should first be investigated. It is generally considered that organic matter plays a key role in the deposition of PAC. Therefore, the effect of the organics species (HA, BSA, and SA) on the deposition of PAC is also investigated in this section.

\subsubsection{Deposition of PAC on the membrane in the absence of organic matter}

The deposition of PAC itself on three types of flat sheet UF membrane (PES, CA, and PVDF) and a hollow fiber UF membrane (PVC) is presented in Fig. 2. For all the membrane, there was a certain amount of virgin PAC $\left(0.26-1.48 \mathrm{~g} / \mathrm{m}^{2}\right)$ irreversibly deposited on their surfaces. Similar to the results of the previous study [29], the deposited PAC did not show measurable impacts on the filtration resistance of the membrane (data not shown). 


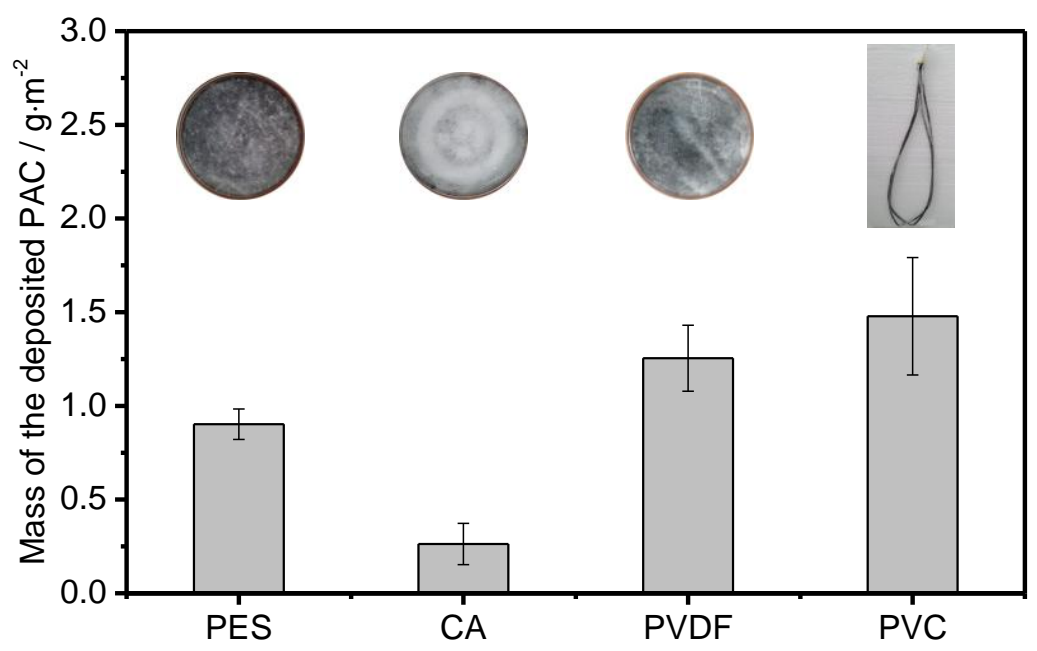

Fig. 2 Deposition of PAC itself on different types of UF membrane. (a) Flat sheet PES membrane; (b) flat sheet CA membrane; (c) flat sheet PVDF membrane; (d) hollow fiber PVC membrane. PAC dosage $=50 \mathrm{mg} / \mathrm{L}$; filtration volume $=300 \mathrm{~mL}$.

The influence of filtration volume on the deposition of PAC itself was also investigated (Fig. S4 of the Supplementary material). The mass of the deposited PAC barely changed as the filtration volume increased. The result suggested that a multilayer could not be formed by the virgin PAC (which was also confirmed by the SEM micrograph in Fig. 6c); thus, the interaction between PAC and the membrane was mainly responsible for the deposition of the PAC itself. Yiantsios and Karabelas [17] investigated the deposition of virgin PAC on a UF membrane when they filtered PAC suspensions in cross-flow mode. They also reported that an equilibrium between the deposition and removal of PAC would be established during filtration.

Because the interaction between the membrane and PAC was the primary factor involved in the deposition of virgin PAC, the properties of the membrane surface 
might affect the deposition of virgin PAC. It is generally accepted that particles readily deposit on membranes with a hydrophobic and rough surface [23]. The contact angles of the CA, PES, PVC, and PVDF membrane were 33.1, 58.2, 81.2, and 85.4 respectively. Therefore, for the virgin PAC, the mass of the deposited PAC increased as the hydrophobicity of the membrane increased.

\subsubsection{Deposition of PAC on the membrane in the presence of different types of organic matter}

The deposition of PAC in the presence of three types of organic matter (i.e., 10 $\mathrm{mg} / \mathrm{L} \mathrm{HA}, 2 \mathrm{mg} / \mathrm{L} \mathrm{BSA}$, and $2 \mathrm{mg} / \mathrm{L} \mathrm{SA}$ ) is shown in Fig. 3. The results showed that the organic matter had a profound influence on the deposition of PAC. The mass of the deposited PAC also varied with different concentrations of calcium, which can change the characteristics and behavior of the organic matter [23].

In the absence of calcium, the mass of the deposited PAC was decreased by HA $\left(0.65 \mathrm{~g} / \mathrm{m}^{2}, p=0.029<0.05\right)$ and SA $\left(0.41 \mathrm{~g} / \mathrm{m}^{2}, p=0.003<0.05\right)$. Therefore, organic matter did not always promote the deposition of PAC. There were two possible reasons for this unexpected phenomenon. (1) The zeta potential of virgin PAC was $-35.7 \mathrm{mV}$, but the zeta potential of the PAC was negatively shifted after adsorbing HA and SA (-43.1 and $-41.5 \mathrm{mV}$, respectively). Consequently, the electrical repulsion between the membrane and the PAC increased. (2) Part of the HA and SA was firstly deposited on the membrane surface, resulting in a steric repulsion effect, and hindered the deposition of PAC [17]. However, the mass of deposited PAC was rarely influenced by BSA $\left(1.01 \mathrm{~g} / \mathrm{m}^{2}, p=0.41>0.05\right)$. The zeta potential of the PAC 
after adsorbing BSA positively shifted to $-29.6 \mathrm{mV}$. As a result, the electrical repulsion between the membrane and the PAC decreased, which may counteract the steric repulsion effect caused by the deposition of BSA on the membrane surface.

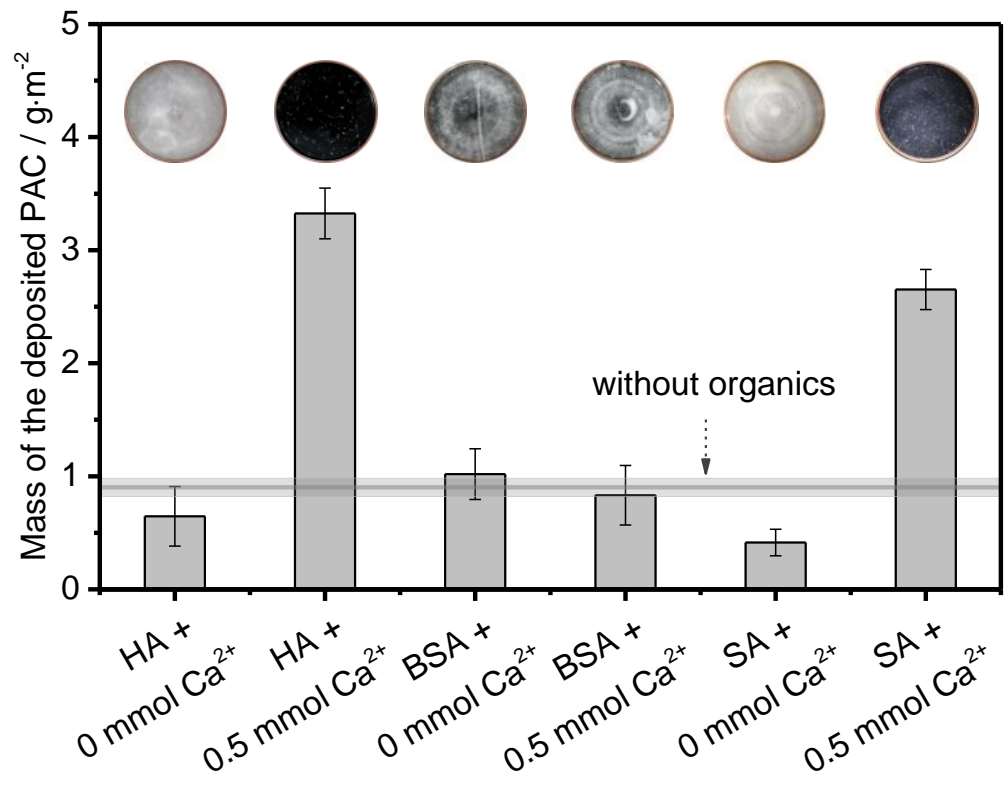

Fig. 3 Deposition of PAC on the PES membrane surface in the presence of humic acid (HA, $10 \mathrm{mg} / \mathrm{L}$ ), bovine serum albumin (BSA, $2 \mathrm{mg} / \mathrm{L}$ ), and sodium alginate (SA, 2 $\mathrm{mg} / \mathrm{L}) . \mathrm{PAC}$ dosage $=50 \mathrm{mg} / \mathrm{L}$, filtration volume $=300 \mathrm{~mL}, \mathrm{Ca}^{2+}$ concentration $=0$ or $0.5 \mathrm{mmol} / \mathrm{L}$.

In the presence of $0.5 \mathrm{mmol} / \mathrm{L}$ calcium ions, the mass of the deposited PAC was dramatically increased by HA $\left(3.33 \mathrm{~g} / \mathrm{m}^{2}, p=7.71 \mathrm{E}-5<0.05\right)$ and SA $\left(2.65 \mathrm{~g} / \mathrm{m}^{2}, p=\right.$ $1.02 \mathrm{E}-4<0.05)$. The results could be due to the following reasons: (1) calcium ions prompted the adsorption of HA and SA on the PAC and membrane surface [10, 30]; (2) the mass of the deposited HA and SA increased in the presence of calcium ions [28, 
31]; (3) the characteristics of the deposited HA and SA changed in the presence of calcium ions. Calcium ions have a strong affinity with the carboxylic moieties of HA, and is able to bridge HA molecules together [23]. Therefore, HA readily deposits on the membrane surface, and forms a "cross-linked" structure. Similar to HA, SA can form complexes with calcium ions, resulting in an "egg-box" structure [32]. The mechanism of how the organic matter promotes the deposition of PAC will be elaborated in detail in Section 3.3. In the presence of $2 \mathrm{mg} / \mathrm{L} \mathrm{BSA}$, the mass of the deposited PAC hardly changed $\left(0.83 \mathrm{~g} / \mathrm{m}^{2}, p=0.67>0.05\right)$.

Comparing all the cases in Fig. 2 and Fig. 3, it could be concluded that in the absence of organic matter, PAC itself was barely deposited on the membrane surface (forming a monolayer); however, in the presence of $\mathrm{HA}$ or $\mathrm{SA}$ with $0.5 \mathrm{mmol} / \mathrm{L}$ calcium ions, the mass of the deposited PAC dramatically increased. Hereafter, the two cases identified (HA or SA with $0.5 \mathrm{mmol} / \mathrm{L}$ calcium ions) were investigated in terms of influencing factors and mechanisms of the deposition of PAC.

\subsection{Factors influencing the deposition of PAC}

\subsubsection{Membrane material}

Three types of flat sheet membrane (PES, CA, and PVDF) were used to study the influence of the membrane material on the deposition of PAC on the UF membrane surface (Fig. 4a). For both HA and SA, the mass of deposited PAC on the different membranes was almost identical $(p>0.05)$. This result is different from the result of virgin PAC in Section 3.1.1. It is generally believed that the membrane material 
significantly affects the deposition of membrane foulants because the interaction between the membrane and the foulants is involved in the deposition of foulants $[6$, 23]. However, the membrane material had a limited effect on the deposition of PAC. A possible explanation for this phenomenon was that the HA and SA in the cake layer, rather than the membrane material, exerted a substantial impact on the deposition of PAC.

a

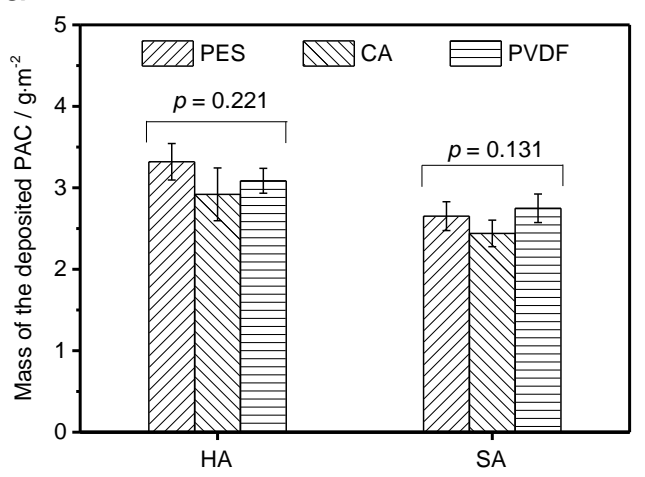

C

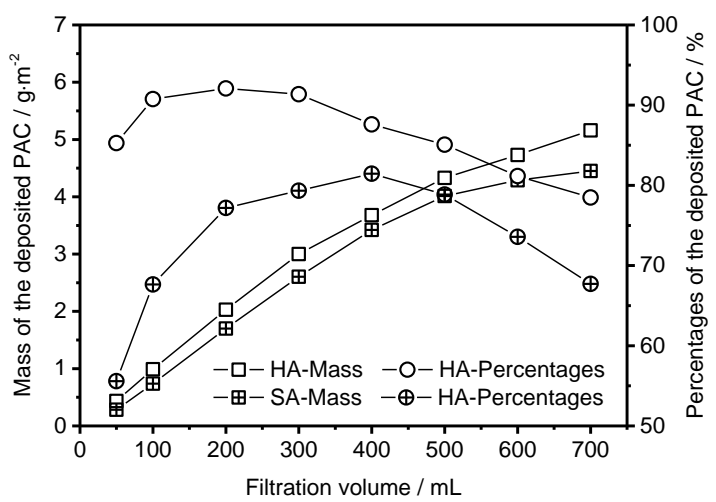

b

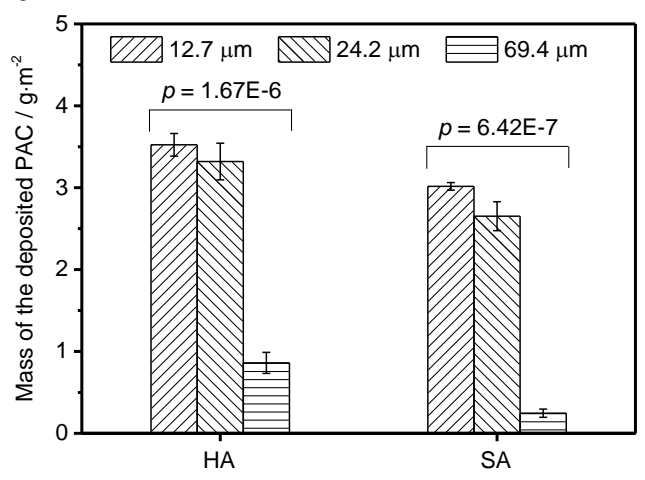

d

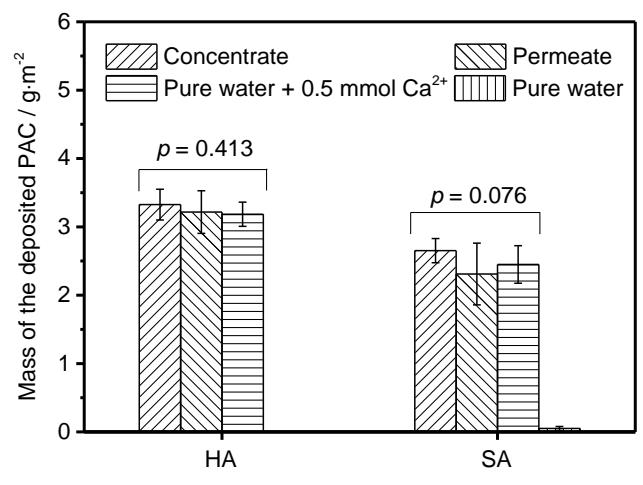

Fig. 4 Factors influencing the deposition of PAC on the UF membrane surface. (a)

Effects of membrane material (PES, CA, and PVDF); (b) effects of PAC size; (c) effects of filtration volume; (d) effects of the water used in physical cleaning. HA concentration $=10 \mathrm{mg} / \mathrm{L}, \mathrm{SA}$ concentration $=2 \mathrm{mg} / \mathrm{L}, \mathrm{Ca}^{2+}=0.5 \mathrm{mmol} / \mathrm{L}, \mathrm{PAC}$ 


$$
\text { dosage }=50 \mathrm{mg} / \mathrm{L} \text {. }
$$

\subsubsection{PAC size}

The deposition of PAC of different sizes $(12.7,24.2$, and $69.4 \mu \mathrm{m})$ on the UF membrane is presented in Fig. 4b. The mass of the deposited PAC decreased as the PAC size increased, both for HA and SA $(p<0.05)$. For example, when $300 \mathrm{~mL}$ of the mixture of $\mathrm{HA}$ and PAC was filtered, the mass of the deposited PAC was $3.52 \mathrm{~g} / \mathrm{m}^{2}$ for the $12.7 \mu \mathrm{m}$ PAC, but the value decreased to $0.86 \mathrm{~g} / \mathrm{m}^{2}$ for the $69.4 \mu \mathrm{m}$ PAC. Compared with the small PAC particles, the large PAC particles are more readily detached by physical cleaning because a stronger shear force is generally applied to the large particles during physical cleaning [33]. Meanwhile, compared with the small PAC, it is harder for the organic matter to play a role during the deposition of large particles of PAC.

\subsubsection{Filtration volume}

The effect of filtration volume on the deposition of PAC on the UF membrane surface is shown in Fig. 4c. As was expected, the mass of the deposited PAC increased as the filtration volume increased (a multilayer was formed). However, the percentage of the irreversibly deposited PAC to the PAC that the membrane received first increased, and then decreased with increasing of filtration volume. The decrease in the percentage of the deposited PAC can be ascribed to the fact that the shear force increased as the thickness of the cake layer increased (the distance between the cake 
layer and the stir bar decreased).

\subsubsection{Chemical compounds of physical cleaning water}

Fig. 4d compares the mass of deposited PAC after physical cleaning (stirring) using various types of physical cleaning water, including UF concentrate, UF permeate, $\mathrm{CaCl}_{2}$ solution $\left(0.5 \mathrm{mmol} / \mathrm{L} \mathrm{Ca}^{2+}\right)$, and Milli-Q water. For both $\mathrm{HA}$ and SA, the mass of deposited PAC was almost identical after physical cleaning using UF concentrate, UF permeate, and $\mathrm{CaCl}_{2}$ solution $(p>0.05)$. By contrast, almost no PAC was detected after physical cleaning using Milli-Q water. By comparing these cases, it can be inferred that calcium ions played an important role in the physical cleaning. Generally, calcium ions can bridge HA molecules together, and the HA shows a "cross-linked" structure on the membrane surface, leading to a compact and stable fouling layer [34]. Similar to HA, SA can also form complexes with calcium ions, and resulting in an "egg-box" structure [35]. When the calcium-free water was used during the physical cleaning, the calcium ions were released from the cake layer, thus destroying the "cross-linked" structure or the "egg-box" structure [28, 31]. As a result, the HA and SA in the cake layer could be removed by physical cleaning using calcium-free water, and thus the PAC could be readily detached.

\subsection{Mechanisms involved in the deposition of PAC}

In Section 3.2, it was found that HA and SA had similar behavior during the deposition of PAC; therefore, only HA was investigated to examine and discuss the mechanisms involved in the deposition of PAC. It was clearly demonstrated that the 
HA in the cake layer promoted the deposition of PAC. The HA in the cake layer could be divided into two parts: (1) HA adsorbed by the PAC and membrane, and (2) HA rejected by the membrane. To identify the role of each of these two parts of HA in the deposition of PAC, a series of experiments was carried out (Table 1).

Table 1 Mass of the deposited PAC under different experimental conditions

\begin{tabular}{|c|c|c|}
\hline $\begin{array}{l}\text { Experiment } \\
\text { No. }\end{array}$ & $\begin{array}{c}\text { Experimental protocol } \\
(\mathrm{HA}=10 \mathrm{mg} / \mathrm{L}, \mathrm{PAC}=50 \mathrm{mg} / \mathrm{L})\end{array}$ & $\begin{array}{l}\text { Mass of the deposited PAC } \\
\qquad\left(\mathrm{g} / \mathrm{m}^{2}\right)\end{array}$ \\
\hline A & Filtered $300 \mathrm{~mL}$ PAC itself & $0.90 \pm 0.08$ \\
\hline B & Filtered $300 \mathrm{~mL}$ "HA + PAC" mixture & $3.33 \pm 0.22$ \\
\hline $\mathrm{C}$ & $\begin{array}{l}\text { Filtered } 300 \mathrm{~mL} \text { "HA (pre-filtered using a } 100 \mathrm{kDa} \\
\text { membrane) + PAC" mixture }\end{array}$ & $0.74 \pm 0.05$ \\
\hline $\mathrm{D}$ & First filtered $300 \mathrm{~mL}$ PAC, then filtered $300 \mathrm{~mL}$ HA & $3.08 \pm 0.37$ \\
\hline $\mathrm{E}$ & First filtered $300 \mathrm{~mL} \mathrm{HA}$, then filtered $300 \mathrm{~mL}$ PAC & $\sim 0$ \\
\hline
\end{tabular}

\subsubsection{The role of the HA adsorbed by the PAC and membrane}

In Experiment $\mathrm{C}$ in Table 1, the HA was pre-filtered with a $100 \mathrm{kDa} \mathrm{CA}$ membrane to remove the HA that could be rejected by the UF membrane. In this case, the HA could be adsorbed by the PAC and membrane, but could not be rejected by the membrane. The results showed that the mass of the HA-laden PAC deposited on the membrane was $0.74 \mathrm{~g} / \mathrm{m}^{2}$, which was lower than the mass of virgin PAC deposited on the membrane $\left(0.90 \mathrm{~g} / \mathrm{m}^{2}, p=0.032<0.05\right)$. The data indicated that the HA adsorbed by the PAC and membrane decreased the mass of deposited PAC. 
Table 2 Total free energy of adhesion $\left(\triangle G^{T O T}\right)$ between PAC particles and between PAC and UF membrane before and after HA adsorption.

\begin{tabular}{ccccc}
\hline \multicolumn{2}{c}{ Free adhesion energies $\left(\mathrm{mJ} / \mathrm{m}^{2}\right)$} & $\Delta G^{L W}$ & $\Delta G^{A B}$ & $\Delta G^{T O T}$ \\
\hline \multirow{2}{*}{ PAC-membrane } & Before HA adsorption & -6.26 & -43.17 & -49.43 \\
& After HA adsorption & -6.48 & -29.01 & -35.49 \\
\multirow{2}{*}{ PAC-PAC } & Before HA adsorption & -9.67 & -14.34 & -24.01 \\
& After HA adsorption & -6.99 & -1.09 & -8.08 \\
\hline
\end{tabular}

The adsorption of HA onto PAC and the UF membrane surface may change the surface properties of the PAC and membrane, and hence change their interactions [23, 36]. The free energy of adhesion was used to quantify the interactions between PAC, HA, and the UF membrane. The parameters of the surface tension components illustrated that the membrane had high electron-donor components, whereas the PAC had high electron-acceptor components (Table S2 of the Supplementary material). After HA adsorption, the hydrophilicity of both the PAC and membrane increased, as indicated by the fact that the polar $\mathrm{AB}$ component of the surface tension increased [26]. The free energy of adhesion of PAC-PAC and PAC-membrane before and after HA adsorption is shown in Table 2. The negative values of the free adhesion energies in Table 2 indicated that the interactions of PAC-PAC and PAC-membrane were attractive forces $[23,26]$. After HA adsorption, the value of $\left|\Delta G^{T O T}\right|$ between the PAC and the membrane decreased from 49.43 to $35.49 \mathrm{~mJ} / \mathrm{m}^{2}$, suggesting that the attractive force between PAC and membrane had decreased. Similarly, the attractive force 
between PAC and PAC also decreased. The reduction of the attractive force could explain the decrease of PAC deposited in Experiment $\mathrm{C}$ in Table 1.

\subsubsection{The role of the HA rejected by the membrane}

Compared with the experiment using unfiltered HA (Experiment B in Table 1, in which the rejected HA was presented), the experiment using HA pre-filtered with a $100 \mathrm{kDa}$ membrane (Experiment $\mathrm{C}$, no rejected HA) dramatically reduced the amount of deposited PAC $\left(0.74 \mathrm{~g} / \mathrm{m}^{2}, p<0.05\right)$. Therefore, HA rejected by the membrane might play a more important role in the deposition of PAC. In Experiment D in Table 1, when the PAC was filtered first, and then the HA was filtered, $3.08 \mathrm{~g} / \mathrm{m}^{2} \mathrm{PAC}$ was deposited on the membrane. However, when the HA was filtered first (Experiment E in Table 1), almost no PAC was deposited on the membrane. In Experiment E, the rejected HA was under the PAC layer; whereas in Experiment D, the rejected HA and PAC formed a cake layer together. The difference suggested that when the PAC and the rejected HA formed a cake layer together, the mass of deposited PAC significantly increased. 


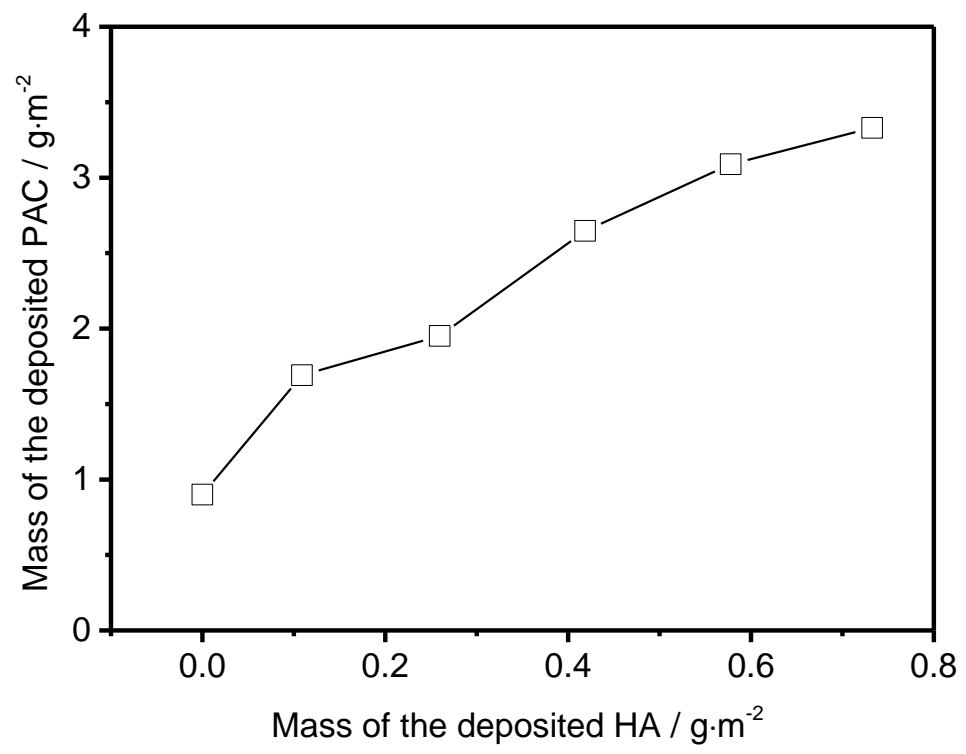

Fig. 5 Relationship between the mass of deposited PAC and the mass of rejected HA on the membrane surface.

The relationship between the mass of deposited PAC and the mass of rejected HA is presented in Fig. 5. The mass of deposited PAC increased as the mass of rejected HA increased. This result confirmed that the rejected HA played a key role in the deposition of PAC.

\subsubsection{Scanning electron microscopy}

The SEM micrograph of the PES membrane surfaces for different cases are shown in Fig. 6. The virgin PES membrane was smooth, and the membrane pores were slightly visible (Fig. 6a). However, for the membrane after HA filtration (0.5 mmol/L $\mathrm{Ca}^{2+}$ ), the cake layer formed by the rejected HA was clearly observed (Fig. 6b). The splitting of the HA cake layer was caused by desiccation during sample preparation. After the filtration of virgin PAC (Fig. 6c), only a small amount of PAC with small 
sizes was irreversibly deposited on the membrane surface. When the mixture of PAC and HA $(<100 \mathrm{kDa})$ was filtered (Fig. 6d), no HA was rejected on the membrane surface, and only some small particles of PAC could be observed on the membrane surface. However, when the mixture of PAC and HA was filtered (Fig. 6e), the rejected HA could be clearly observed (splitting of the HA cake layer was observed), and a large amount of PAC was "trapped" in the HA layer.
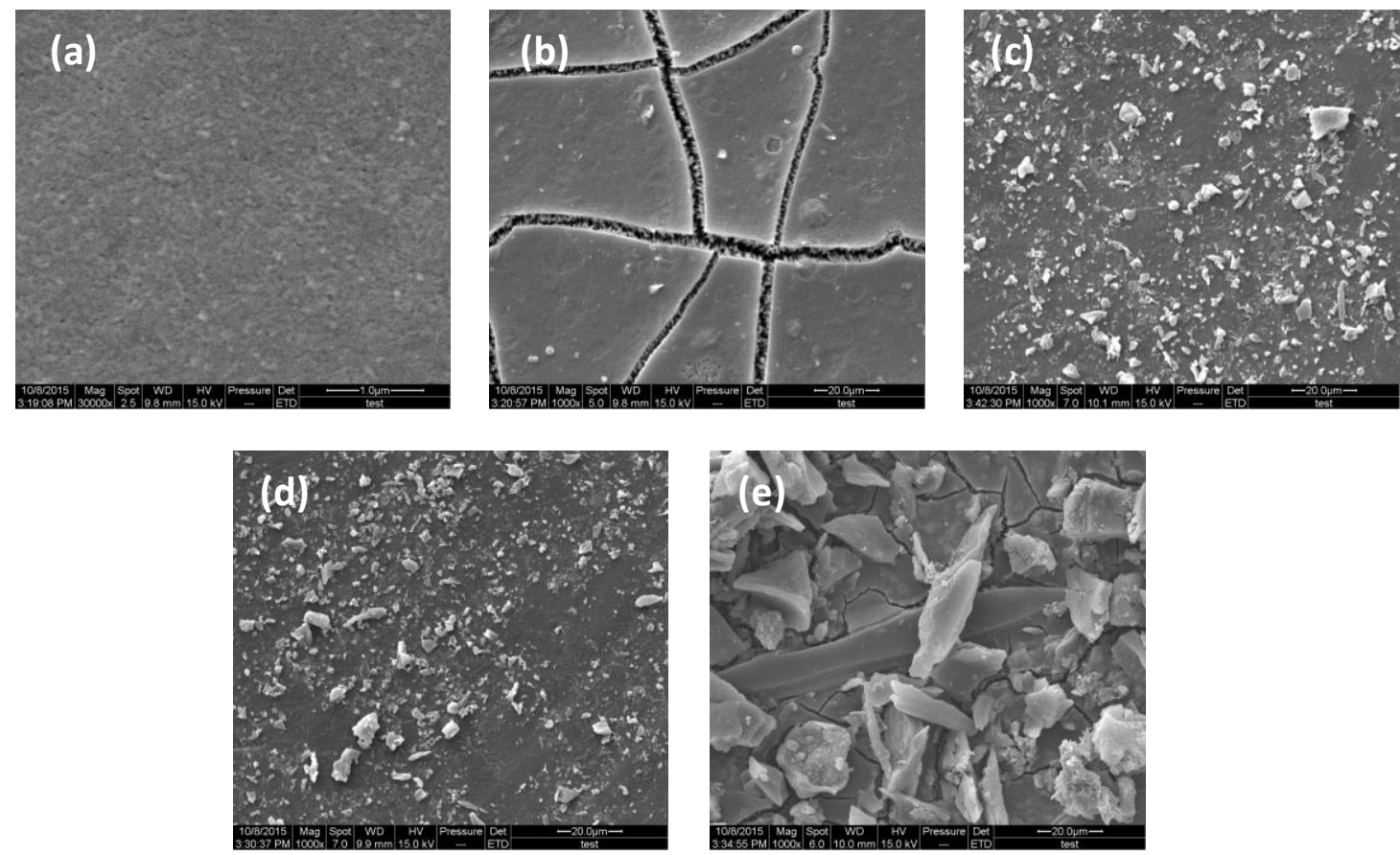

Fig. 6 SEM pictures of the PES membrane surface after physical cleaning. (a) Virgin membrane surface (×30000); (b) filtration of $300 \mathrm{~mL} 10 \mathrm{mg} / \mathrm{L} \mathrm{HA}(\times 1000)$; (c) filtration of PAC itself $(\times 1000)$; (d) filtration of $300 \mathrm{~mL} \mathrm{HA}$ (pre-filtered, $<100 \mathrm{kDa}$ ) and PAC mixture (×1000); (e) filtration of $300 \mathrm{~mL} \mathrm{HA}(10 \mathrm{mg} / \mathrm{L})$ and PAC (50 mg/L) mixture $(\times 1000)$. 


\subsubsection{Mechanisms involved in the deposition of PAC}

A schematic of the mechanisms involved in the deposition of PAC on the UF membrane surface is summarized in Fig. 7. For the virgin PAC, a multilayer could not be formed, therefore the virgin PAC was hardly deposited on the membrane surface. After adsorbing HA, the attractive force between the PAC and the membrane decreased; thus, the HA adsorbed by the membrane and PAC could not "glue" the PAC on the membrane surface and therefore hindered the deposition of PAC. When the PAC and the HA rejected by the membrane formed a cake layer together, the mass of deposited PAC dramatically increased. Two possible reasons might be responsible for the increase. (1) The deposited HA had a relatively high contact area with the membrane and PAC (because the HA can change its shape during the deposition), compared with the contact areas of PAC-PAC and PAC-membrane. Consequently, the attractive force between PAC and the deposited HA was relatively high. (2) The deposited HA could fill the spaces between PAC and PAC as well as PAC and membrane, and the PAC could be "trapped" in the HA layer (Fig. 6e).

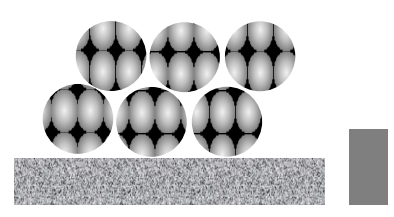

Virgin PAC

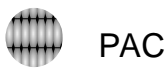

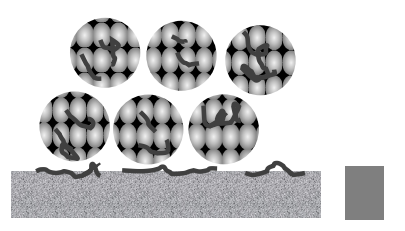

PAC after adsorbing $\mathrm{HA}$

HA

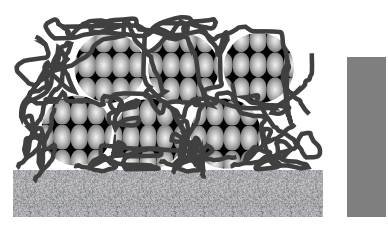

PAC and the deposited HA

Mass of the deposited PAC

Fig. 7 Schematic diagram of the mechanisms involved in the deposition of PAC on the UF membrane surface. 
In Fig. 3, in the absence of calcium ions, it was very hard for the HA and SA to form a stable cake layer [30, 34]; the molecular size of BSA is smaller than the pore size of UF membrane [23], and thus BSA could not be rejected by the membrane. Therefore, PAC was rarely deposited on the membrane surface in these cases. However, in the presence of $0.5 \mathrm{mmol} / \mathrm{L}$ calcium ions, the rejected HA or SA could form a stable cake layer with PAC. Consequently, a large amount of PAC was deposited on the membrane. The results shown in Fig. 4 could also be explained by this mechanism. The deposition of PAC was determined by the deposited organic matter, so the mass of deposited PAC was barely affected by the membrane material. It is more difficult for large PAC to be "trapped" in the HA, therefore the mass of deposited PAC decreased as the PAC size increased. The mass of deposited organic matter increased as the filtration volume increased, and thus the mass of deposited PAC increased with the increase in filtration volume. When cleaning the fouled membrane with pure water, the deposited organic matter could be detached from the membrane surface. Consequently, the deposited PAC could be effectively removed.

The organic matter deposited on the membrane surface was the main reason for the deposition of PAC. Therefore, to reduce the mass of the deposited PAC, the deposition of organic matter should be minimized, especially high molecular-weight organic matter. In other words, the hybrid PAC/UF may be more suitable for the treatment of feed water that mainly contains low molecular-weight organic matter. In 
practical applications, the pretreatment process and physically cleaning are generally used to reduce the deposition of organic matter on the UF membrane surface $[37,38]$. These methods are also effective in reducing the deposition of PAC. During the operation of the hybrid PAC/UF process, we can also select PAC with large sizes and use pure water for backwashing to reduce the deposition of PAC.

\section{Conclusions}

In the hybrid PAC/UF process, the deposition of PAC on the membrane surface was systematically investigated. The following conclusions can be drawn:

(1) In the absence of organic matter, the PAC itself was barely deposited on the membrane surface (forming a monolayer). However, when PAC was filtered with HA or $\mathrm{SA}$ in the presence of calcium ions, the mass of the deposited PAC was dramatically increased, and a multilayer was formed by PAC and organic matter.

(2) The deposition of PAC was rarely influenced by membrane material. The mass of deposited PAC increased as the PAC size decreased and the filtration volume increased. Physical cleaning with pure water could effectively detach the deposited PAC.

(3) The HA adsorbed by the membrane and PAC decreased the attractive force of membrane-PAC and PAC-PAC, therefore hindering the deposition of PAC.

(4) When the PAC and the rejected HA formed a cake layer together, the PAC could be "trapped" in the HA layer. Consequently, the mass of deposited PAC significantly increased. 


\section{Acknowledgements}

This research was jointly supported by the National Natural Science Foundation of China (51608396, 51508423), China Postdoctoral Science Foundation funded projects (Grants 2016M592386), and the National Science Foundation for the Outstanding Youngster Fund (51522804).

\section{Appendix A. Supplementary material}

\section{References}

[1] V.L. Snoeyink, C. C, B.J. Mariñas, Design and performance of powdered activated carbon/ultrafiltration systems, Water Sci. Technol. 42 (2000) 1-10.

[2] C. Stoquart, P. Servais, P.R. Bérubé, B. Barbeau, Hybrid Membrane Processes using activated carbon treatment for drinking water: A review, J. Membr. Sci. 411-412 (2012) 1-12.

[3] S. Lee, J.-W. Lee, S. Kim, P.-K. Park, J.-H. Kim, C.-H. Lee, Removal of $17 \beta$-estradiol by powdered activated carbon-Microfiltraion hybrid process: The effect of PAC deposition on membrane surface, J. Membr. Sci. 326 (2009) 84-91.

[4] C. Tansakul, S. Laborie, C. Cabassud, Adsorption combined with ultrafiltration to remove organic matter from seawater, Water Res. 45 (2011) 6362-6370.

[5] W. Yu, L. Xu, J. Qu, N. Graham, Investigation of pre-coagulation and powder activate carbon adsorption on ultrafiltration membrane fouling, J. Membr. Sci. 459 (2014) 157-168.

[6] K. Li, H. Liang, F. Qu, S. Shao, H. Yu, Z.-s. Han, X. Du, G. Li, Control of 
natural organic matter fouling of ultrafiltration membrane by adsorption pretreatment: Comparison of mesoporous adsorbent resin and powdered activated carbon, J. Membr. Sci. 471 (2014) 94-102.

[7] C. Stoquart, P. Servais, B. Barbeau, Ammonia removal in the carbon contactor of a hybrid membrane process, Water Res. 67 (2014) 255-266.

[8] S. Shao, F. Qu, H. Liang, K. Li, H. Yu, H. Chang, G. Li, Powdered activated carbon - membrane bioreactor operated under intermittent aeration and short sludge retention times for micro-polluted surface water treatment, Int. Biodeterior. Biodegrad. 102 (2015) 81-88.

[9] S. Shao, H. Liang, F. Qu, H. Yu, K. Li, G. Li, Fluorescent natural organic matter fractions responsible for ultrafiltration membrane fouling: Identification by adsorption pretreatment coupled with parallel factor analysis of excitation-emission matrices, J. Membr. Sci. 464 (2014) 33-42.

[10] S. Shao, H. Liang, F. Qu, K. Li, H. Chang, H. Yu, G. Li, Combined influence by humic acid (HA) and powdered activated carbon (PAC) particles on ultrafiltration membrane fouling, J. Membr. Sci. 500 (2016) 99-105.

[11] G. Seo, S. Jang, S. Lee, C. Yoon, The fouling characterization and control in the high concentration PAC membrane bioreactor HCPAC-MBR, Water Sci. Technol. $51(2005) 77-84$.

[12] S. Shao, F. Qu, H. Liang, H. Chang, H. Yu, G. Li, A pilot-scale study of a powdered activated carbon-membrane bioreactor for the treatment of water with a 
high concentration of ammonia, Environ. Sci.: Water Res. Technol. 2 (2016) 125-133.

[13] M.M. Zhang, C. Li, M.M. Benjamin, Y.J. Chang, Fouling and natural organic matter removal in adsorbent/membrane systems for drinking water treatment, Environ. Sci. Technol. 37 (2003) 1663-1669.

[14] H. Oh, M. Yu, S. Takizawa, S. Ohgaki, Evaluation of PAC behavior and fouling formation in an integrated PAC-UF membrane for surface water treatment, Desalination 192 (2006) 54-62.

[15] S.-K. Kang, K.-H. Choo, Why does a mineral oxide adsorbent control fouling better than powdered activated carbon in hybrid ultrafiltration water treatment?, J. Membr. Sci. 355 (2010) 69-77.

[16] S. Leveille, A. Carriere, S. Charest, B. Barbeau, PAC membrane bioreactor as an alternative to biological activated carbon filters for drinking water treatment, J. Water Supply: Res. Technol. - AQUA 62 (2013) 23-33.

[17] S.G. Yiantsios, A. Karabelas, An experimental study of humid acid and powdered activated carbon deposition on UF membranes and their removal by backwashing, Desalination 140 (2001) 195-209.

[18] P. Zhao, S. Takizawa, H. Katayama, S. Ohgaki, Factors causing PAC cake fouling in PAC-MF (powdered activated carbon-microfiltration) water treatment systems, Water Sci. Technol. 51 (2005) 231-240.

[19] F. Saravia, F.H. Frimmel, Role of NOM in the performance of adsorption-membrane hybrid systems applied for the removal of pharmaceuticals, 
Desalination 224 (2008) 168-171.

[20] M. Campinas, M.J. Rosa, Assessing PAC contribution to the NOM fouling control in PAC/UF systems, Water Res. 44 (2010) 1636-1644.

[21] E. Filloux, H. Gallard, J.-P. Croue, Identification of effluent organic matter fractions responsible for low-pressure membrane fouling, Water Res. 46 (2012) $5531-5540$.

[22] H.C. Kim, B.A. Dempsey, Membrane fouling due to alginate, SMP, EfOM, humic acid, and NOM, J. Membr. Sci. 428 (2013) 190-197.

[23] C.Y. Tang, T.H. Chong, A.G. Fane, Colloidal interactions and fouling of NF and RO membranes: A review, Adv. Colloid Interface Sci. 164 (2011) 126-143.

[24] F. Zamani, A. Ullah, E. Akhondi, H.J. Tanudjaja, E.R. Cornelissen, A. Honciuc, A.G. Fane, J.W. Chew, Impact of the surface energy of particulate foulants on membrane fouling, J. Membr. Sci. 510 (2016) 101-111.

[25] H. Cao, O. Habimana, A.J.C. Semião, A. Allen, R. Heffernan, E. Casey, Understanding particle deposition kinetics on NF membranes: A focus on micro-beads and membrane interactions at different environmental conditions, J. Membr. Sci. 475 (2015) 367-375.

[26] N. Subhi, A.R.D. Verliefde, V. Chen, P. Le-Clech, Assessment of physicochemical interactions in hollow fibre ultrafiltration membrane by contact angle analysis, J. Membr. Sci. 403-404 (2012) 32-40.

[27] Q. Wang, Z. Wang, C. Zhu, X. Mei, Z. Wu, Assessment of SMP fouling by 
foulant-membrane interaction energy analysis, J. Membr. Sci. 446 (2013) 154-163.

[28] H. Chang, F. Qu, B. Liu, H. Yu, K. Li, S. Shao, G. Li, H. Liang, Hydraulic irreversibility of ultrafiltration membrane fouling by humic acid: Effects of membrane properties and backwash water composition, J. Membr. Sci. 493 (2015) 723-733.

[29] F. Saravia, C. Zwiener, F.H. Frimmel, Influence of PAC properties on membrane performance in a PAC-UF hybrid system, Water Sci. Tech. - W. Sup. 12 (2012) 496-503.

[30] P. van den Brink, A. Zwijnenburg, G. Smith, H. Temmink, M. van Loosdrecht, Effect of free calcium concentration and ionic strength on alginate fouling in cross-flow membrane filtration, J. Membr. Sci. 345 (2009) 207-216.

[31] H. Chang, H. Liang, F. Qu, S. Shao, H. Yu, B. Liu, W. Gao, G. Li, Role of backwash water composition in alleviating ultrafiltration membrane fouling by sodium alginate and the effectiveness of salt backwashing, J. Membr. Sci. 499 (2016) 429-441.

[32] G.T. Grant, E.R. Morris, D.A. Rees, P.J.C. Smith, D. Thom, Biological interactions between polysaccharides and divalent cations: The egg-box model, FEBS Letters 32 (1973) 195-198.

[33] H. Li, A.G. Fane, H.G.L. Coster, S. Vigneswaran, Direct observation of particle deposition on the membrane surface during crossflow microfiltration, J. Membr. Sci. 149 (1998) 83-97.

[34] I. Sutzkover-Gutman, D. Hasson, R. Semiat, Humic substances fouling in 
ultrafiltration processes, Desalination 261 (2010) 218-231.

[35] S. Meng, Y. Liu, Ultrafiltration behaviors of alginate blocks at various calcium concentrations, Water Res. 83 (2015) 248-257.

[36] D. Jermann, W. Pronk, M. Boller, Mutual influences between natural organic matter and inorganic particles and their combined effect on ultrafiltration membrane fouling, Environ. Sci. Technol. 42 (2008) 9129-9136.

[37] A.W. Zularisam, A.F. Ismail, R. Salim, Behaviours of natural organic matter in membrane filtration for surface water treatment - A review, Desalination 194 (2006) 211-231.

[38] H. Huang, K. Schwab, J.G. Jacangelo, Pretreatment for low pressure membranes in water treatment: A review, Environ. Sci. Technol. 43 (2009) 3011-3019. 


\section{Graphical abstract}

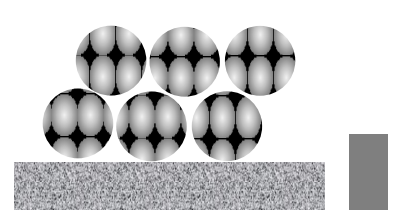

Virgin PAC

(11.1!) PAC

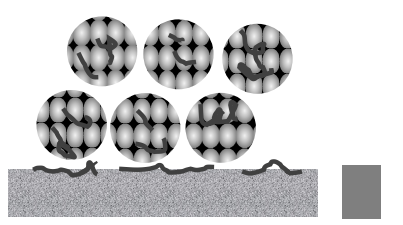

PAC after adsorbing $H A$

HA

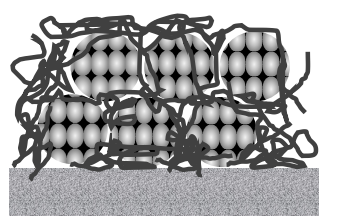

$\mathrm{PAC}$ and the deposited HA

Mass of the deposited PAC 